\title{
OPEN The occurrence of aflatoxins and human health risk estimations in randomly obtained maize from some markets in Ghana
}

\author{
Nii Korley Kortei ${ }^{1 \bowtie}$, Theophilus Annan², Papa Toah Akonor ${ }^{3}$, Seidu A. Richard ${ }^{4}$, \\ Helen Ama Annan ${ }^{3}$, Vincent Kyei-Baffour ${ }^{5}$, Felicia Akuamoa ${ }^{6}$, Princess Golda Akpaloo ${ }^{1}$ \& \\ Paul Esua-Amoafo ${ }^{1}$
}

Maize and its products are most often prone to fungal contamination especially during cultivation and storage by toxigenic fungi. Aflatoxicosis still persist in Ghana despite the numerous education on several ways of its prevention at the farm as well as its adverse health implications which are food safety concerns. A random assessment and human risk analysis was conducted on 90 maize (72 white and 18 colored) samples from markets across all the regions of Ghana. Total aflatoxins (AFtotal) and the constitutive aflatoxins $\left(\mathrm{AFB}_{1}, \mathrm{AFB}_{2}, \mathrm{AFG}_{1}\right.$, and $\mathrm{AFG} \mathrm{G}_{2}$ ) were analyzed by High-Performance Liquid Chromatography (HPLC). Out of a total of ninety (90) samples investigated, $72(80 \%)$ tested positive for $\mathrm{AFB}_{1}$ and the contamination levels ranged from $0.78 \pm 0.04$ to $339.3 \pm 8.6 \mathrm{\mu g} \mathrm{kg}^{-1}$. Similarly, AFG2 was detected in only $14(15.5 \%)$ samples, and their values ranged between $1.09 \pm 0.03$ and $5.51 \pm 0.26 \mu \mathrm{g} \mathrm{kg}^{-1}$ while AF total ranged between $0.78 \pm 0.04$ and $445.01 \pm 8.9 \mu \mathrm{g} \mathrm{kg}^{-1}$ constituting approximately $72(80 \%)$. Limits of $\mathrm{AFB}_{1}$ and total aflatoxins (AFtotal) for the Ghana Standards Authority (GSA) (5 and $10 \mu \mathrm{g} \mathrm{kg}^{-1}$ ) and the European Food Safety Authority (EFSA) (2 and $4 \mathrm{\mu g} \mathrm{kg}^{-1}$ ), were used as checks. A total of $33(41.25 \%)$ samples were above the limits for both. Risk assessments recorded for Estimated Daily Intake (EDI), Hazard Quotient (H.Q), Hazard Index (H.I), Margin of Exposure (MOE), av. Potency, and population risks ranged $0.087-0.38 \mu \mathrm{g} \mathrm{kg}^{-1}$ bw day ${ }^{-1}, 1.5-6.9$, $0.0087-0.38,3.64-12.09,0-0.0396 \mathrm{ng}^{-1}$ Alatoxins $\mathrm{kg}^{-1} \mathrm{bw} \mathrm{day}^{-1}$ and, $3.5 \times 10^{-1}-0.015$ respectively for total aflatoxins. While ranges for aflatoxins B1 (AFB1) recorded were $0.068-0.3 \mu \mathrm{g} \mathrm{Kg} \mathrm{bw}^{-1} \mathrm{day}^{-1}$, 2.43-10.64, 0.0068-0.030, 4.73-20.51, 0-0.0396 ng Aflatoxins $\mathrm{kg}^{-1} \mathrm{bw} \mathrm{day}^{-1}$ and, $2.69 \times 10^{-3}-0.012$ for Estimated Daily Intake (EDI), Hazard Quotient (H.Q), Hazard Index (H.I), Margin of Exposure (MOE), Av. potency, and population risks respectively. It was deduced that although there was some observed contamination of maize across the different ecological zones, the consumption of maize (white and colored) posed no adverse health effects on the population of Ghana since computed $\mathrm{H}$.I was less than $1(<1)$.

Zea mays (Maize) is a principal cereal and staple for people living in warm climates throughout Asia, Africa, and the Americas who are predisposed to the effects of climate change ${ }^{1}$ in terms of production, consumption and income generation. Forming part of everyday meals, maize, and its products since time immemorial, has been part of the African culture, and so form part of an everyday meal in most homes ${ }^{2}$. It is commonly consumed fresh or processed into cooked or fermented, milled and beverage products ${ }^{3-5}$. It is also extensively used to prepare delectable dishes either singly or in combination with other staples particularly groundnuts or legumes or animal sources of protein to complement each other to combat malnutrition since its protein content is inadequate $e^{23}$.

\footnotetext{
${ }^{1}$ Department of Nutrition and Dietetics, School of Allied Health Sciences, University of Health and Allied Sciences, PMB 31, Ho, Ghana. ${ }^{2}$ Food Microbiology Division, Council for Scientific and Industrial Research- Food Research Institute, P. O. Box M20, Accra, Ghana. ${ }^{3}$ Food Processing and Engineering Division, Council for Scientific and Industrial Research- Food Research Institute, P. O. Box M20, Accra, Ghana. ${ }^{4}$ Department of Medicine, Princefield University, P.O. Box MA 128, Ho, Ghana. ${ }^{5}$ Food Chemistry and Nutrition Research Division, Council for Scientific and Industrial Research- Food Research Institute, P. O. Box M20, Accra, Ghana. ${ }^{6}$ Applied Radiation Biology Centre, Biotechnology and Nuclear Agriculture Research Institute, Ghana Atomic Energy Commission, P. O. Box AE 1, Atomic, Accra, Ghana. ${ }^{\boxplus e m a i l: ~ n k k o r t e i @ u h a s . e d u . g h ~}$
} 
In Ghana, maize is broadly appreciated as a stable crop since it is grown in all agro-ecological zones. More than $50 \%$ of rural households cultivate it traditionally under rainfed conditions. Besides, also $16 \%$ of urban households are involved in its production. However, there is a yield gap especially in the northern and upper regions. This has ultimately created an imbalance between its production and consumption ${ }^{6}$. Intake levels of approximately $43-46 \mathrm{~kg}_{\text {person }}{ }^{-1}$ day $^{-1}$ of household consumption of maize in rural subsistence farming communities in Ghana have been reported ${ }^{7,8}$.

Maize is susceptible to fungal infections mainly from Fusarium and Aspergillus species and consequent contamination with their mycotoxins; fumonisins and aflatoxins ${ }^{9}$ respectively throughout its growth, harvest, transport, and storage ${ }^{10,11}$. A change in climate simultaneously impacts the complex communities of Aflatoxin (AF)-producing fungi by altering the number of AF-producers to change its fungal community's structure.

Aflatoxins are secondary metabolites, which are naturally occurring contaminants of food and elaborate the toxins under auspicious conditions of temperature, relative humidity, and poor storage conditions. They are now known to be mainly produced by A. flavus, A. parasiticus, Aspergillus nomius and two different Emericella species $^{12}$.

Biochemically, aflatoxins are difurano-coumarin derivatives with a bifuran group joined to the coumarin nucleus and a pentanone ring (in case of AFBs) or a lactone ring (in case of AFGs) ${ }^{13,14}$. AFB1, AFB2, AFG1, and AFG2 are the four most significant AFs among the identified 20. The B-types are produced by A. flavus while G-types are produced by A. parasiticus ${ }^{15}$. Yu et al. ${ }^{16}$, as well as Yabe and Nakajimam ${ }^{17}$ identified approximately 18 enzymatic steps with at least 25 genes answerable for producing the enzymes and regulating the biosynthesis of aflatoxins process. All the aflatoxins-producing fungi exhibit a great variation in terms of qualitative and quantitative differences in the toxicology abilities that are noticeable attributes by different strains within each fungal species.

Consequently, in sub-Saharan Africa, mycotoxin studies have focused mostly on aflatoxins (in maize and groundnuts) and fumonisins (in maize) while the other potentially dangerous mycotoxins in other foods have received less attention. This is possibly so because maize is a staple food with extensive use that complements groundnuts to combat protein-energy malnutrition typically used in complementary feeding ${ }^{18}$ and so naturally any microorganism and toxins that affect it directly, will be of critical concern. All valuations of exposure implicate maize or groundnuts as the main source of aflatoxins or fumonisins.

The total maize harvest in Africa according to FAO (2017), was estimated at 40 million hectares, with Nigeria being the top producer (16\%) followed by Tanzania. Worldwide maize consumption is estimated to be more than 116 million tons with $30 \%$ and $21 \%$ of the consumption occurring globally and in Sub-Saharan African (SSA), respectively. Approximately 14 countries in SSA consume $85-95 \%$ of white maize as their staple food ${ }^{19}$. In most of the developing countries from Africa, there is an increased risk of hepatocellular carcinoma in the presence of hepatitis B virus infection and esophageal cancer being linked to aflatoxins contamination of food ${ }^{18}$.

As emphasized by some previous researchers ${ }^{20-23}$, mycotoxins especially aflatoxins toxicity has always been a topic of contentious interest in the international market and economic development of a country, many of agricultural products are often rejected due to excessive contaminations (beyond specific thresholds of host countries). This is evidenced in previously published works on aflatoxins and cereals in Ghana which revealed some tenacity and unsatisfactory trend of contamination ${ }^{24-28}$.

To overcome this problem, many countries have set standard safety levels of aflatoxins in food and food products and animal feed to ensure quality. Furthermore, several attempts have been made to educate the populace and stakeholders on the preventive practices and impacts of these mycotoxins on health ${ }^{29}$ chiefly attributable to non-compliance to Good Management Practices (GMP), Good Agricultural Practices (GAP) and Good Hygienic Practice (GHP).

It was hypothesized that maize grains meant for consumption and sold on markets across Ghana did not contain aflatoxins. The objective of this study was, therefore, to assess the potential exposure to aflatoxin through consumption of commercial maize products (market maize), we conducted a cross-sectional assessment of market maize contamination.

\section{Materials and methods}

Study area. On the Gulf of Guinea in West Africa, is located in Ghana. It covers about 23,884,245 ha of land and water area between latitudes $4^{\circ} \mathrm{N}$ and $11^{\circ} \mathrm{N}$ and longitudes $4^{\circ} \mathrm{W}$ and $2^{\circ} \mathrm{E}^{30,31}$. The country is demarcated into 10 regions and 216 districts, categorized into five main agro-ecological (Coastal Savannah, Evergreen, Deciduous Forest, Transitional, and Savannah) zones (Fig. 1). An estimated 24,658,832 people were counted across the whole country during the 2010 Ghana Population and Housing Census ${ }^{32}$.

The three northern regions (Upper West, Upper East, and Northern) are predominantly agro-ecologically Savannah. Ashanti, Brong-Ahafo, and Eastern regions are mainly transitional and deciduous forest areas. The western region is mostly Evergreen and Deciduous Forest and highly economically active. Greater Accra (predominantly Coastal) and Ashanti (predominantly Deciduous Forest) are the most developed and urbanized regions and the lowest in terms of agricultural activity. The Volta region cuts across three agro-ecological (coastal, deciduous forest, and savannah) zones. The central region, primarily deciduous forest and coastal, is the fourth poorest region. Fishing and agriculture are the main economic activities. The five principal maize growing areas are in the Northern, Brong-Ahafo, Ashanti, Central and Eastern Regions.

Sample collection. To collect a representative data set, we first obtained the list of villages in each district from the Regional Directorate of the Ministry of Agriculture. From each district, an average of 9 villages (Table 1) were then randomly selected. The maize sellers in each market were conveniently sampled where about one kilogram $(1 \mathrm{~kg})$ of raw maize samples were purchased concurrently within the period of February to 


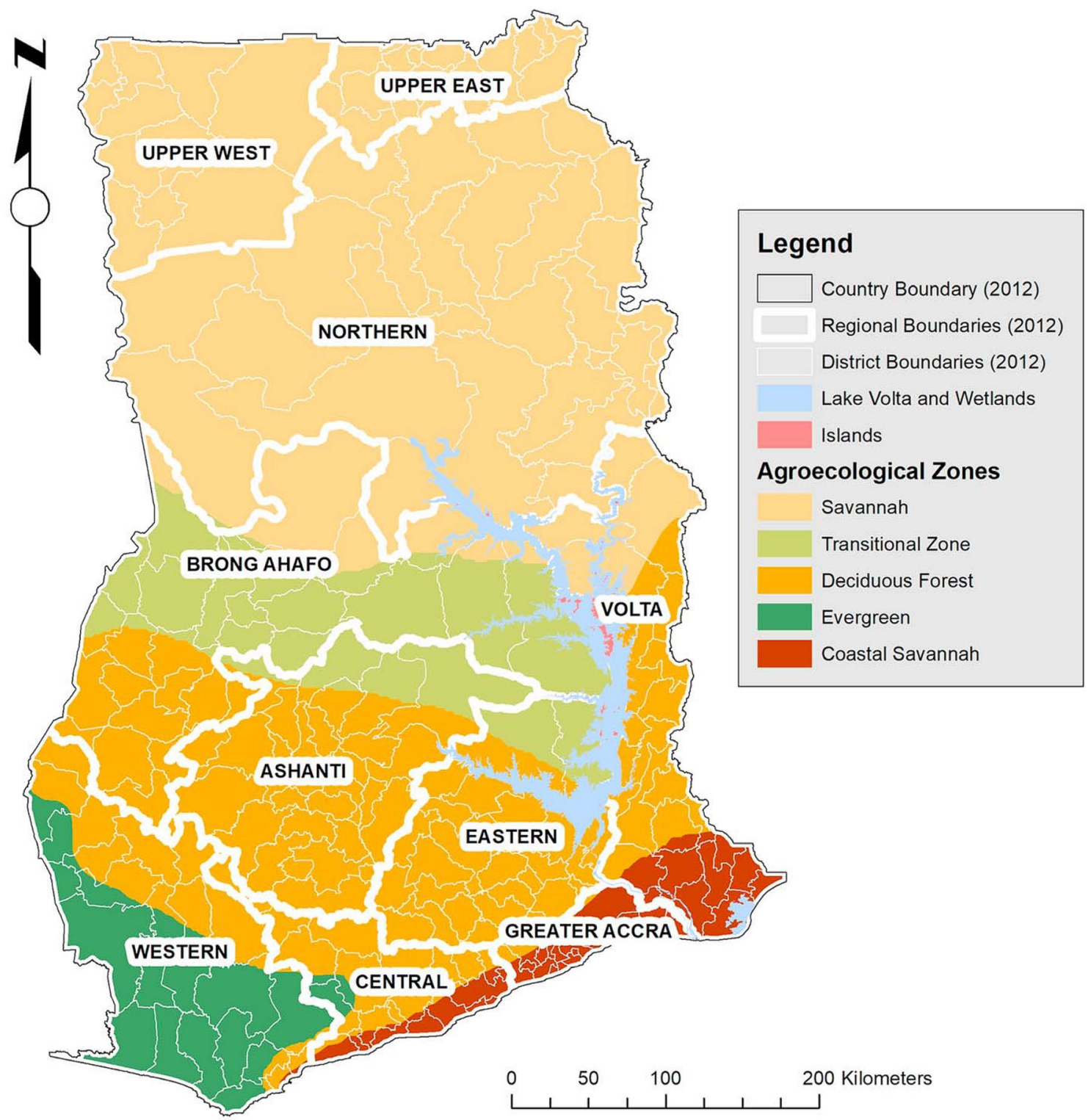

Figure 1. Map of Ghana showing regions of sampling (Adapted from Abbam et al. ${ }^{33}$ ).

\begin{tabular}{|l|l|l|l|l|l|}
\hline Region & No. of samples & Agro-ecological zones & Rainfall $(\mathbf{m m})$ & Temperature $\left({ }^{\circ} \mathbf{C}\right)$ & Coordinates \\
\hline Greater Accra & $9 / 90$ & Coastal Savannah & $800-1000$ & 26.6 & $5.8143^{\circ} \mathrm{N}, 0.0747^{\circ} \mathrm{E}$ \\
\hline Central & $9 / 90$ & Deciduous Forest & $1400-1600$ & 26.7 & $5.5608^{\circ} \mathrm{N}, 1.0586^{\circ} \mathrm{W}$ \\
\hline Western & $8 / 90$ & Evergreen & $1800-2000$ & 25.9 & $5.3902^{\circ} \mathrm{N}, 2.1450^{\circ} \mathrm{W}$ \\
\hline Eastern & $12 / 90$ & Deciduous Forest & $1400-1900$ & 25.9 & $6.2374^{\circ} \mathrm{N}, 0.4502^{\circ} \mathrm{W}$ \\
\hline Ashanti & $11 / 90$ & Deciduous Forest & $1200-1400$ & 26.3 & $6.7470^{\circ} \mathrm{N}, 1.5209^{\circ} \mathrm{W}$ \\
\hline Brong-Ahafo & $9 / 90$ & Transitional zone & $1400-1600$ & 23.9 & $7.9559^{\circ} \mathrm{N}, 1.6761^{\circ} \mathrm{W}$ \\
\hline Volta & $7 / 90$ & Coastal Savannah/Deciduous forest & $1000-1400$ & 26.2 & $6.5781^{\circ} \mathrm{N}, 0.4502^{\circ} \mathrm{E}$ \\
\hline Northern & $10 / 90$ & Savannah & $1000-1200$ & 27.9 & $9.5439^{\circ} \mathrm{N}, 0.9057^{\circ} \mathrm{W}$ \\
\hline Upper East & $7 / 90$ & Savannah & $800-1000$ & 28.3 & $10.7082^{\circ} \mathrm{N}, 0.9821^{\circ} \mathrm{W}$ \\
\hline Upper West & $8 / 90$ & Savannah & $1000-1200$ & 27.8 & $10.2530^{\circ} \mathrm{N}, 2.1450^{\circ} \mathrm{W}$ \\
\hline
\end{tabular}

Table 1. Geographical locations and some attributes of the origin of samples. 


\begin{tabular}{|l|l|l|}
\hline Aflatoxin & Limits & Amount $(\boldsymbol{\mu g} / \mathbf{k g})$ \\
\hline \multirow{2}{*}{ AFB1 } & LOD & 0.20 \\
\cline { 2 - 3 } & LOQ & 0.60 \\
\hline \multirow{2}{*}{ AFB2 } & LOD & 0.17 \\
\cline { 2 - 3 } & LOQ & 0.51 \\
\hline \multirow{2}{*}{ AFG1 } & LOD & 0.26 \\
\cline { 2 - 3 } & LOQ & 0.78 \\
\hline \multirow{2}{*}{ AFG2 } & LOD & 0.36 \\
\cline { 2 - 3 } & LOQ & 1.08 \\
\hline
\end{tabular}

Table 2. Limits of Detection and Quantification (LOD \& LOQ) of aflatoxins $\mathrm{AFB}_{1}, \mathrm{AFB}_{2}, \mathrm{AFG}_{1}, \mathrm{AFG}_{2}$ and Total aflatoxins $(\mu \mathrm{g} / \mathrm{kg})$ measured by HPLC. $L O D$ limit of detection, $L O Q$ limit of quantification.

\begin{tabular}{|c|c|c|c|c|c|c|}
\hline \multirow[b]{2}{*}{ Category } & \multirow[b]{2}{*}{ Food sample } & \multicolumn{5}{|c|}{ Concentrations of Aflatoxins $\left(\mu \mathrm{g} \mathrm{kg}^{-1}\right)$} \\
\hline & & AFB1 & AFB2 & AFG1 & AFG2 & Total \\
\hline \multirow{18}{*}{ White } & MzHalf- Assini & $59.5 \pm 0.99^{\mathrm{a}}$ & $10.9 \pm 0.4^{\mathrm{a}}$ & n.d & n.d & $70.4 \pm 1.8^{\mathrm{a}}$ \\
\hline & MzDzodze & $8.77 \pm 0.47^{\mathrm{g}}$ & $2.40 \pm 0.3^{\mathrm{d}}$ & $0.98 \pm 0.03^{\mathrm{d}}$ & n.d & $12.15 \pm 1.1^{\mathrm{h}}$ \\
\hline & MzKaneshie & $16.02 \pm 1.2^{\mathrm{e}}$ & $2.53 \pm 0.15^{\mathrm{dc}}$ & n.d & n.d & $18.55 \pm .0 .9^{\mathrm{g}}$ \\
\hline & MzKsi cm & $9.65 \pm 0.25^{\mathrm{g}}$ & $2.98 \pm 0.92^{\mathrm{c}}$ & $2.71 \pm 0.91^{\mathrm{bc}}$ & n.d & $15.34 \pm 0.9^{\text {gh }}$ \\
\hline & MzSuhum & $16.27 \pm 0.39^{\mathrm{e}}$ & $3.11 \pm 0.5^{c}$ & $1.89 \pm 0.05^{\mathrm{d}}$ & $1.97 \pm 0.06^{\mathrm{a}}$ & $23.24 \pm 0.32 \mathrm{f}$ \\
\hline & MzKintamp & $20.4 \pm 0.8^{\mathrm{d}}$ & $4.93 \pm 0.27^{\mathrm{bc}}$ & $3.51 \pm 0.85 b$ & $1.66 \pm 0.06^{\mathrm{a}}$ & $30.5 \pm 2.15^{\mathrm{dc}}$ \\
\hline & MzMoree & $8.03 \pm 1.7^{\mathrm{g}}$ & $0.93 \pm 0.04^{\mathrm{e}}$ & n.d & n.d & $8.96 \pm 0.22^{\mathrm{i}}$ \\
\hline & MzKorle G & n.d & n.d & n.d & n.d & n.d \\
\hline & MzNsawam & $4.92 \pm 0.6^{\mathrm{h}}$ & $0.69 \pm 0.03^{\mathrm{e}}$ & n.d & n.d & $5.61 \pm 0.26^{\mathrm{i}}$ \\
\hline & $\mathrm{MzHo} \mathrm{cm}$ & n.d & n.d & n.d & n.d & n.d \\
\hline & MzWa & n.d & n.d & n.d & n.d & n.d \\
\hline & MzKotokraba & $5.31 \pm 0.26^{\mathrm{h}}$ & $0.86 \pm 0.03^{\mathrm{e}}$ & n.d & n.d & $6.17 \pm 0.8^{i}$ \\
\hline & MzAowin & $47.75 \pm 2.25^{\mathrm{b}}$ & $5.23 \pm 0.25^{b}$ & $2.98 \pm 0.9^{\mathrm{b}}$ & n.d & $55.96 \pm 1.2^{\mathrm{b}}$ \\
\hline & MzSunyani & $34.32 \pm 2.1^{\mathrm{c}}$ & $5.2 \pm 0.22^{\mathrm{b}}$ & n.d & n.d & $39.55 \pm 2.3^{\mathrm{dc}}$ \\
\hline & MzMadina & $14.92 \pm 0.5^{\mathrm{ef}}$ & $1.5 \pm 0.07^{\mathrm{de}}$ & $2.31 \pm 0.91^{b c}$ & n.d & $18.73 \pm 0.65^{g}$ \\
\hline & MzAsafo & $42.07 \pm 1.5^{\mathrm{b}}$ & $3.17 \pm 0.15^{c}$ & n.d & n.d & $45.24 \pm 1.45^{c}$ \\
\hline & MzBerekum & $5.18 \pm 0.25^{\mathrm{h}}$ & $0.67 \pm 0.02^{\mathrm{e}}$ & n.d & n.d & $5.85 \pm 0.26^{\mathrm{i}}$ \\
\hline & MzTema & $11.17 \pm 1.22 \mathrm{f}$ & $10.24 \pm 1.02^{\mathrm{a}}$ & $5.6 \pm 0.55^{\mathrm{a}}$ & n.d & $27.01 \pm 0.4^{\mathrm{e}}$ \\
\hline
\end{tabular}

Table 3. Concentration of aflatoxin types in different maize samples from different locations of Ghana. Mean \pm Standard Deviation that do not share a letter are significantly different $(p>0.05)$. n.d not detected.

September 2018 and grouped into 2 categories (white and colored maize) (Tables 3, 4, 5, 6 and 7) Twenty (20) grams each of maize samples were fetched and kept in sterile bags in ice chests and sent to the laboratory within the same day in a vehicle where they were stored in a deep freezer at $-20^{\circ} \mathrm{C}$ until ready for chemical analysis ${ }^{35}$.

Extraction of samples. $\quad \mathrm{AFB}_{1}, \mathrm{AFB}_{2}, \mathrm{AFG}$, and $\mathrm{AFG}_{2}$ were extracted from samples according to the European Committee for Standardization (CEN) official method EN14123 $3^{34}$ for aflatoxin extraction. Methanol in water $(200 \mathrm{ml})(8+2)$ and $5 \mathrm{~g} \mathrm{NaCl}$ were used to extract $25 \mathrm{~g}$ of sample. Hexane $(100 \mathrm{ml})$ was added to samples containing more than $50 \%$ fat. Mixture was homogenized for $3 \mathrm{~min}$ at $3000 \mathrm{rpm}$ ( $2 \mathrm{~min})$ and $3500 \mathrm{rpm}(1 \mathrm{~min})$. The extracts were filtered and $10 \mathrm{ml}$ of filtrate added to $60 \mathrm{ml}$ of phosphate buffer saline (PBS) for solid-phase extraction using a pre-conditioned immune-affinity columns specific for $\mathrm{AFB}_{1}, \mathrm{AFB}_{2}, \mathrm{AFG}_{1}$, and $\mathrm{AFG}_{2}$. The $70 \mathrm{ml}$ filtrate-PBS mixture was loaded onto the pre-conditioned column and allowed to elute by gravity at a flow rate of $1 \mathrm{ml} \mathrm{min}^{-1}$. This was followed by a cleanup with $15 \mathrm{ml}$ distilled water at a flow rate of $5 \mathrm{ml} \mathrm{min}^{-1}$. Aflatoxins were eluted in two steps into a $5 \mathrm{ml}$ volumetric flask with $0.5 \mathrm{ml}$ followed by $0.75 \mathrm{ml}$ of methanol (HPLC grade) and allowed to elute by gravity. Deionized water was used to make up volume of eluates to $5 \mathrm{ml}$ and eluate vortexed and $2 \mathrm{ml}$ pipetted into HPLC vials for quantification ${ }^{35}$.

HPLC parameters. Injection volume: $10 \mu \mathrm{l}$ flow rate: $1 \mathrm{ml} \mathrm{min}{ }^{-1}$, column temperature: $35^{\circ} \mathrm{C}$, excitation wavelength: $360 \mathrm{~nm}$, emission wavelength: $440 \mathrm{~nm}$, mobile phase composition: water/acetonitrile/MeOH (65:15:20 v/v/v), post-column derivatization: Kobra cells. HPLC Column Specification Spherisorb ODS1- Excel $(4.6 \mathrm{~mm} \times 25 \mathrm{~cm}), 5 \mu \mathrm{m}$ particle size, 250 A pore size.

LOD $=$ Limit of detection. 


\begin{tabular}{|c|c|c|c|c|c|c|}
\hline \multirow[b]{2}{*}{ Category } & \multirow[b]{2}{*}{ Food sample } & \multicolumn{5}{|c|}{ Concentrations of Aflatoxins $(\mu \mathrm{g} / \mathrm{kg})$} \\
\hline & & AFB1 & AFB2 & AFG1 & AFG2 & Total \\
\hline \multirow{18}{*}{ White } & MzKoforidua & n.d & n.d & n.d & n.d & n.d \\
\hline & MzWalewale & $12.87 \pm 1.4^{\mathrm{c}}$ & $0.61 \pm 0.05^{\mathrm{e}}$ & n.d & n.d & $13.48 \pm 0.35^{\mathrm{c}}$ \\
\hline & MzNsawkaw & $5.29 \pm 0.24^{\mathrm{de}}$ & n.d & $2.51 \pm 0.14^{\mathrm{b}}$ & n.d & $7.8 \pm 0.45^{\mathrm{e}}$ \\
\hline & MzAsamankese & $7.44 \pm 1.2^{\mathrm{d}}$ & $4.86 \pm 0.44^{\mathrm{b}}$ & $4.22 \pm 0.2^{\mathrm{a}}$ & $2.06 \pm 0.17^{\mathrm{a}}$ & $18.58 \pm 1.1^{\mathrm{c}}$ \\
\hline & MzHohoe & $14.4 \pm 1.26^{c}$ & $0.56 \pm 0.03^{\mathrm{e}}$ & n.d & n.d & $14.96 \pm 1.3^{\mathrm{d}}$ \\
\hline & MzAbutia & $7.07 \pm 0.32^{\mathrm{d}}$ & $0.52 \pm 0.02^{\mathrm{e}}$ & n.d & n.d & $7.53 \pm 0.50^{\mathrm{e}}$ \\
\hline & MzSogakope & $12.92 \pm 1.2^{\mathrm{c}}$ & $5.0 \pm 0.42^{\mathrm{b}}$ & $4.2 \pm 0.26^{\mathrm{a}}$ & n.d & $22.12 \pm 0.45^{\circ}$ \\
\hline & MzKintampo & n.d & n.d & n.d & n.d & n.d \\
\hline & MzTamale & $44.20 \pm 0.51^{\mathrm{a}}$ & $7.7 \pm 0.50^{\mathrm{a}}$ & $1.6 \pm 0.05^{\mathrm{bc}}$ & n.d & $53.5 \pm 0.95^{\mathrm{a}}$ \\
\hline & MzKayera & n.d & n.d & n.d & n.d & n.d \\
\hline & MzNanton1 & $10.63 \pm 1.2^{c}$ & n.d & n.d & n.d & $10.63 \pm 1.20^{\mathrm{c}}$ \\
\hline & MzNanton 2 & $11.42 \pm 1.22^{c}$ & $1.19 \pm 0.1^{\mathrm{d}}$ & n.d & n.d & $12.61 \pm 1.20^{\mathrm{c}}$ \\
\hline & MzSavelugu & $22.33 \pm 0.35^{b}$ & $4.75 \pm 0.48^{\mathrm{bc}}$ & n.d & n.d & $27.08 \pm 1.4^{\mathrm{b}}$ \\
\hline & MzAssin fosu & $20.73 \pm 0.3^{b}$ & $4.75 \pm 0.48^{\mathrm{bc}}$ & n.d & n.d & $25.08 \pm 1.4^{\mathrm{b}}$ \\
\hline & MzAdeamra & n.d & n.d & n.d & n.d & n.d \\
\hline & MzAfrancho & n.d & n.d & n.d & n.d & n.d \\
\hline & MzEjisu.mkt & $8.55 \pm 0.60^{\mathrm{d}}$ & n.d & n.d & n.d & $8.55 \pm 0.60^{\mathrm{e}}$ \\
\hline & MzAfrmso & $4.96 \pm 0.43^{\mathrm{e}}$ & n.d & $2.09 \pm 0.13^{\mathrm{b}}$ & n.d & $7.05 \pm 0.50^{\mathrm{e}}$ \\
\hline
\end{tabular}

Table 4. Concentration of aflatoxin types in different maize samples from different locations of Ghana. Mean \pm Standard Deviation that do not share a letter are significantly different $(\mathrm{p}>0.05)$. n.d not detected.

\begin{tabular}{|c|c|c|c|c|c|c|}
\hline \multirow[b]{2}{*}{ Category } & \multirow[b]{2}{*}{ Food sample } & \multicolumn{5}{|c|}{ Concentrations of Aflatoxins $(\mu \mathrm{g} / \mathrm{kg})$} \\
\hline & & AFB1 & AFB2 & AFG1 & AFG2 & Total \\
\hline \multirow{18}{*}{ White } & MzDonkork & $60.51 \pm 1.65^{\mathrm{c}}$ & $17.66 \pm 1.5^{c}$ & $10.21 \pm 1.1^{\mathrm{b}}$ & $2.7 \pm 0.6^{\mathrm{c}}$ & $91.08 \pm 2.1^{\mathrm{b}}$ \\
\hline & MzKwaekese & $7.86 \pm 0.45^{\mathrm{h}}$ & $0.54 \pm 0.04^{\mathrm{g}}$ & n.d & n.d & $8.4 \pm 0.85^{\mathrm{g}}$ \\
\hline & MzPedu & n.d & n.d & n.d & n.d & n.d \\
\hline & MzAfrm.pl & $14.28 \pm 1.20^{\mathrm{g}}$ & $1.58 \pm 0.06^{\mathrm{g}}$ & $13.19 \pm 0.9^{\mathrm{a}}$ & n.d & $29.05 \pm 3.50 f$ \\
\hline & MzKayeru & n.d & n.d & n.d & n.d & n.d \\
\hline & MzKamina & $2.5 \pm 0.12^{\mathrm{i}}$ & n.d & $4.53 \pm 0.22^{\mathrm{d}}$ & n.d & $7.03 \pm 0.4^{\mathrm{g}}$ \\
\hline & MzAgbogbloshi & $45.13 \pm 0.61^{\mathrm{d}}$ & $16.32 \pm 1.3^{\mathrm{c}}$ & $11.5 \pm 1.22^{\mathrm{b}}$ & $2.8 \pm 0.12^{\mathrm{c}}$ & $75.75 \pm 1.84^{\mathrm{c}}$ \\
\hline & MzNima & $39.5 \pm 3.01^{\mathrm{de}}$ & $13.77 \pm 0.9^{d}$ & $4.99 \pm 0.26^{\mathrm{d}}$ & $5.5 \pm 0.27^{\mathrm{a}}$ & $63.76 \pm 1.71^{d}$ \\
\hline & MzMakola & $94.42 \pm 2.24^{b}$ & n.d & n.d & n.d & $94.42 \pm 2.24^{\mathrm{b}}$ \\
\hline & \begin{tabular}{|l|} 
MzZuo \\
\end{tabular} & $1.88 \pm 0.06^{\mathrm{i}}$ & n.d & n.d & n.d & $1.88 \pm 0.06^{\mathrm{h}}$ \\
\hline & MzZebilla & $2.26 \pm 0.6 \mathrm{i}$ & n.d & n.d & n.d & $2.26 \pm 0.6^{\mathrm{h}}$ \\
\hline & MzMankesim & $23.11 \pm 0.31 f$ & $8.3 \pm 0.5 f$ & $4.67 \pm 0.47^{\mathrm{d}}$ & n.d & $36.08 \pm 2.5^{\mathrm{e}}$ \\
\hline & MzSandema & $43.7 \pm 2.25^{\mathrm{d}}$ & $21.6 \pm 0.33^{b}$ & $13.75 \pm 1.2^{\mathrm{a}}$ & n.d & $79.05 \pm 1.82^{c}$ \\
\hline & MzNkwatia & $232.47 \pm 8.33^{\mathrm{a}}$ & $15.77 \pm 1.4^{\mathrm{c}}$ & n.d & n.d & $248.24 \pm 8.45^{\mathrm{a}}$ \\
\hline & MzDromkma & n.d & n.d & n.d & n.d & n.d \\
\hline & MzFunsi & $36.4 \pm 2.5^{\mathrm{de}}$ & $20.5 \pm 0.35^{\mathrm{b}}$ & $6.8 \pm 0.33^{\mathrm{cd}}$ & $1.11 \pm 0.05^{\mathrm{d}}$ & $64.8 \pm 1.67^{\mathrm{d}}$ \\
\hline & MzAbliri & $25.2 \pm 0.35 f$ & $11.7 \pm 1.2^{\mathrm{de}}$ & n.d & n.d & $36.9 \pm 2.14^{\mathrm{e}}$ \\
\hline & MzBielepong & $51.73 \pm 0.3^{\mathrm{d}}$ & $24.1 \pm 0.4^{\mathrm{a}}$ & $14.5 \pm 1.2^{\mathrm{a}}$ & $3.5 \pm 0.6^{\mathrm{b}}$ & $93.83 \pm 5.2^{\mathrm{b}}$ \\
\hline
\end{tabular}

Table 5. Concentration of aflatoxin types in different maize samples from different locations of Ghana. Mean \pm Standard Deviation that do not share a letter are significantly different $(\mathrm{p}>0.05)$. n.d not detected.

LOQ = Limit of quantification.

$\mathrm{ACN}=$ Acetonitrile.

$\mathrm{MeOH}=$ Methanol.

LOD calculation $=3 *$ standard deviation/slope.

LOQ calculation $=3 \times$ LOD.

Supplier of Column R- Biopharm, Block 10 campus, West Scotland.

Science Park, Acre Road, Glasgow, Scotland G20 OXA ${ }^{35}$. 


\begin{tabular}{|c|c|c|c|c|c|c|}
\hline \multirow[b]{2}{*}{ Category } & \multirow[b]{2}{*}{ Food Sample } & \multicolumn{5}{|c|}{ Concentrations of Aflatoxins $(\mu \mathrm{g} / \mathrm{kg})$} \\
\hline & & AFB1 & AFB2 & AFG1 & AFG2 & Total \\
\hline \multirow{18}{*}{ White } & MzBakano & n.d & n.d & n.d & n.d & n.d \\
\hline & MzUCC & $2.2 \pm 0.15^{\mathrm{i}}$ & n.d & n.d & n.d & $2.2 \pm 0.15^{j}$ \\
\hline & MzAdul & n.d & n.d & n.d & n.d & n.d \\
\hline & MzAdu2 & n.d & n.d & n.d & n.d & n.d \\
\hline & MzEjura & $339.3 \pm 8.6^{\mathrm{a}}$ & $103 \pm 2.5^{\mathrm{a}}$ & $2.71 \pm 0.12^{\mathrm{cd}}$ & n.d & $445.01 \pm 8.9^{\mathrm{a}}$ \\
\hline & MzBonwire & $102.1 \pm 2.5^{c}$ & $0.88 \pm 0.03^{\mathrm{de}}$ & n.d & n.d & $102.98 \pm 2.5^{\mathrm{d}}$ \\
\hline & MzSefw. W & $11.5 \pm 0.3^{\mathrm{g}}$ & $0.62 \pm 0.03^{\mathrm{de}}$ & n.d & n.d & $12.12 \pm 1.4^{\mathrm{g}}$ \\
\hline & MzBibiani & $5.21 \pm 0.32^{\mathrm{h}}$ & $1.5 \pm 0.06^{\mathrm{d}}$ & n.d & n.d & $6.71 \pm 0.08^{\mathrm{i}}$ \\
\hline & MzSefw.Bk & $185.17 \pm 2.15^{\mathrm{b}}$ & $9.37 \pm 0.25^{\mathrm{b}}$ & n.d & n.d & $194.54 \pm 2.5^{\mathrm{b}}$ \\
\hline & MzAtwim & $18.21 \pm 1.31 \mathrm{f}$ & $2.66 \pm 0.13^{c}$ & n.d & n.d & $20.87 \pm 0.35^{\mathrm{g}}$ \\
\hline & MzT'di & $3.93 \pm 0.22^{\mathrm{h}}$ & $0.79 \pm 0.03^{\mathrm{de}}$ & n.d & n.d & $4.72 \pm 0.28^{\mathrm{i}}$ \\
\hline & MzNsaw & n.d & n.d & n.d & n.d & n.d \\
\hline & MzTumu & $75.4 \pm 1.85^{\mathrm{d}}$ & $40.3 \pm 0.55^{\mathrm{a}}$ & $7.62 \pm 0.33^{b}$ & $2.57 \pm 0.3^{\mathrm{b}}$ & $125.89 \pm 2.5^{c}$ \\
\hline & MzAzuguyeri & $24.7 \pm 0.35^{\mathrm{e}}$ & $6.77 \pm 0.30^{\mathrm{bc}}$ & $2.61 \pm 0.92^{\mathrm{cd}}$ & n.d & $34.08 \pm 3.01 f$ \\
\hline & MzBolga & $5.18 \pm 0.25^{\mathrm{h}}$ & $2.91 \pm 0.9^{c}$ & $1.02 \pm 0.02^{\mathrm{d}}$ & n.d & $9.11 \pm 0.22^{\mathrm{h}}$ \\
\hline & MzKayeru & $25.96 \pm 0.31^{\mathrm{e}}$ & $7.89 \pm 0.32^{b}$ & $1.22 \pm 0.06^{\mathrm{d}}$ & n.d & $35.07 \pm 2.82 \mathrm{f}$ \\
\hline & MzChogu & $10.91 \pm 1.22^{\mathrm{g}}$ & $4.23 \pm 0.23^{c}$ & $2.5 \pm 0.92^{\mathrm{cd}}$ & $1.88 \pm 0.06^{\mathrm{bc}}$ & $19.52 \pm 0.31^{\mathrm{g}}$ \\
\hline & MzOffinso & $27.78 \pm 0.52^{\mathrm{e}}$ & $9.47 \pm 0.46^{\mathrm{b}}$ & $5.41 \pm 0.25^{\mathrm{ab}}$ & $5.51 \pm 0.26^{\mathrm{a}}$ & $48.17 \pm 0.55^{\mathrm{e}}$ \\
\hline
\end{tabular}

Table 6. Concentration of aflatoxin types in different maize samples from different locations of Ghana. Mean \pm Standard Deviation that do not share a letter are significantly different $(\mathrm{p}>0.05)$. n.d not detected.

\begin{tabular}{|c|c|c|c|c|c|c|}
\hline \multirow[t]{2}{*}{ Category } & \multirow[b]{2}{*}{ Food Sample } & \multicolumn{5}{|c|}{ Concentrations of Aflatoxins $(\mu \mathrm{g} / \mathrm{kg})$} \\
\hline & & AFB1 & AFB2 & AFG1 & AFG2 & Total \\
\hline \multirow{18}{*}{ Colored } & MzLawra & $2.69 \pm 0.12^{\mathrm{e}}$ & $1.55 \pm 0.05^{\mathrm{ab}}$ & $1.01 \pm 0.02^{\mathrm{b}}$ & $1.09 \pm 0.03^{\mathrm{a}}$ & $6.29 \pm 0.81^{\mathrm{cd}}$ \\
\hline & MzDambai & $5.35 \pm 0.7^{\mathrm{d}}$ & $1.67 \pm 0.05^{\mathrm{ab}}$ & $1.05 \pm 0.11^{\mathrm{b}}$ & n.d & $8.07 \pm 0.85^{c}$ \\
\hline & MzGbawe & $2.37 \pm 0.12^{\mathrm{e}}$ & n.d & n.d & n.d & $2.37 \pm 0.12^{\mathrm{e}}$ \\
\hline & MzKintampo & $16.95 \pm 0.84^{\mathrm{a}}$ & $1.04 \pm 0.06^{\mathrm{ab}}$ & $3.67 \pm 0.22^{\mathrm{a}}$ & n.d & $21.66 \pm 0.32^{\mathrm{a}}$ \\
\hline & MzGurugu & $1.77 \pm 0.06^{\mathrm{e}}$ & n.d & n.d & n.d & $1.77 \pm 0.06^{\mathrm{e}}$ \\
\hline & MzKeta & $1.61 \pm 0.06^{\mathrm{e}}$ & n.d & n.d & n.d & $1.61 \pm 0.06^{\mathrm{e}}$ \\
\hline & MzTech jxn & $0.88 \pm 0.02^{\mathrm{ef}}$ & $0.67 \pm 0.02^{\mathrm{ab}}$ & n.d & n.d & $1.55 \pm 0.06^{\mathrm{e}}$ \\
\hline & MzGwolu & $1.78 \pm 0.06^{\mathrm{e}}$ & $0.80 \pm 0.03^{\mathrm{ab}}$ & n.d & n.d & $2.66 \pm 0.15^{\mathrm{e}}$ \\
\hline & MzDormaa & $3.01 \pm 0.5^{\mathrm{e}}$ & $2.55 \pm 0.14^{\mathrm{a}}$ & n.d & n.d & $5.56 \pm 0.26^{\mathrm{cd}}$ \\
\hline & MzTechiman & $17.43 \pm 0.81^{\mathrm{a}}$ & $3.2 \pm 0.22^{\mathrm{a}}$ & n.d & n.d & $20.63 \pm 0.33^{\mathrm{a}}$ \\
\hline & MzBame & $5.2 \pm 0.5^{\mathrm{c}}$ & $0.77 \pm 0.04^{\mathrm{ab}}$ & $1.02 \pm 0.05^{\mathrm{b}}$ & n.d & $6.99 \pm 0.82^{c}$ \\
\hline & MzKwadaso & $8.41 \pm 0.85^{b}$ & $2.85 \pm 0.14^{\mathrm{a}}$ & $1.01 \pm 0.05^{\mathrm{b}}$ & n.d & $11.26 \pm 1.22^{\mathrm{b}}$ \\
\hline & MzLapaz & $4.5 \pm 0.27^{\mathrm{d}}$ & $1.6 \pm 0.07^{\mathrm{ab}}$ & $1.07 \pm 0.06^{b}$ & n.d & $7.17 \pm 0.45^{c}$ \\
\hline & MzJuaboso & $1.89 \pm 0.06^{\mathrm{e}}$ & $0.82 \pm 0.04^{\mathrm{ab}}$ & n.d & n.d & $2.71 \pm 0.15^{\mathrm{e}}$ \\
\hline & MzNavrongo-ue & $0.78 \pm 0.04^{\mathrm{ef}}$ & n.d & n.d & n.d & $0.78 \pm 0.04 \mathrm{f}$ \\
\hline & MzMkt circle & n.d & n.d & $1.1 \pm 0.02^{\mathrm{b}}$ & n.d & $1.1 \pm 0.02^{\mathrm{e}}$ \\
\hline & MzAnomabo & n.d & n.d & n.d & n.d & n.d \\
\hline & MzNadowli & n.d & n.d & n.d & n.d & n.d \\
\hline
\end{tabular}

Table 7. Concentration of aflatoxin types in different maize samples from different locations of Ghana. Mean \pm Standard Deviation that do not share a letter are significantly different $(\mathrm{p}>0.05) . n . d$ not detected.

Analysis of samples. The aflatoxins (by Aspergillus flavus and A. parasiticus) levels in the samples were determined by High-Performance Liquid Chromatography HPLC (Agilent 1260 Series, OpenLab software, X-bridge column $)(250 \mathrm{~mm} \times 4.6 \mathrm{~mm}$, i.d., $5 \mu \mathrm{m})$, USA with fluorescence detector and post-column derivatization using Kobra cells to generate bromine electrochemically at the CSIR- Food Research Institute, Ghana. LOD for AFB1, AFB2, AFG1 and AFG2 were $0.20 \mu \mathrm{g} \mathrm{kg}^{-1}, 0.17 \mu \mathrm{g} \mathrm{kg}^{-1}, 0.26 \mu \mathrm{g} \mathrm{kg}{ }^{-1}$ and $0.36 \mu \mathrm{g} \mathrm{kg}^{-1}$ respectively (Table 2$)^{35}$.

Limit of detection/quantification (LOD/LOQ). Limit of detection and quantification (LOD/LOQ) of the HPLC were estimated by making a calibration curve around the least standard used for spiking, $5 \mu \mathrm{kg}^{-1}$ 
(lowest concentration range of calibration curve). Blank did not produce any signal, so the LOD and LOQ were calculated as;

$$
\begin{gathered}
\text { LOD }=3 * \text { standard deviation/slope. } \\
\text { LOQ }=3 * \text { LOD. }
\end{gathered}
$$

Measurement accuracy. Spiking of pure aflatoxin standard solution was done according to method described by Kortei et al. ${ }^{35}$ to ensure measurement accuracy of analysis. Three levels spiking were done at the lower, mid and upper concentration range of the calibration curve concentrations (5 ppb, $15 \mathrm{ppb}$ and $30 \mathrm{ppb}$ ). Spike volumes of pure standards were calculated as;

$$
[\text { Sample weight }(\mathrm{g}) * \text { spike concentration }(\mathrm{ppb})] /[\text { Concentration of standard }(\mathrm{ug} / \mathrm{ml})] \text {. }
$$

Spike volumes were distributed evenly on aflatoxins free sample (blank) and spiked sample analysed for percentage recovery which was calculated as;

[(Concentration measured in spike-concentration measured in blank)/(spiked amount)]*100

Measurement precision. Repeatability and intermediate precision analyses of an internal reference material (IRM) was used to ensure measurement precision of the method. For repeatability analysis, 10 parallel extractions of the IRM was done by the same analyst at the same time using the same HPLC and the relative standard deviation among results calculated. For intermediate precision, 10 extractions of the IRM were done at different days by different analysts and the relative standard deviation among results calculated ${ }^{35}$. The relative standard deviations were calculated as; [Standard deviation/mean] ${ }^{\star} 100$.

Required performance criteria for accuracy and precision. Repeatability. Relative standard deviation among repeatable results should be less than $15 \%$.

Intermediate precision. Relative standard deviation among results obtained under intermediate precision conditions should be less than $20 \%$.

Recovery. Percent recovery of measurement procedure should be in a range of $80-120 \%$.

Limit of detection. The limit of detection should be less than $1 \mathrm{ug} \mathrm{kg}^{-1}$ for all aflatoxins.

Limit of quantification. The limit of Quantification should be less than $3 \mathrm{ug} \mathrm{kg}^{-1}$ for all aflatoxins.

Linearity. Linearity from regression curve should be $0.99(\mathrm{~B} 1, \mathrm{~B} 2, \mathrm{G} 1)$ and $0.98(\mathrm{G} 2)^{35}$.

\section{Experimental data}

Repeatability. Relative standard deviation was; B1 $=5.5 \%$; B2 $=6.7 \%$; G1 $=7.4 \%$; G2 $=12.1 \%$ and Total aflatoxins $=5.2 \%$.

Intermediate Precision (Reproducibility): Relative standard deviation was; B1 =13.2\%; B2 =13.4\%; G1 = 13.7\%; $\mathrm{G} 2=12.2 \%$ and Total aflatoxins $=11.9 \%$

Recovery: Percent recovery of measurement procedure was; Low concentration: $\mathrm{B} 1=107 \%$; $\mathrm{B} 2=87.2 \%$; $\mathrm{G} 1=113.4 \% ; \mathrm{G} 2=112.8 \%$ and Total aflatoxins $=108.2 \%$.

High concentration: $\mathrm{B} 1=102.6 \% ; \mathrm{B} 2=101.6 \% ; \mathrm{G} 1=104.2 \% ; \mathrm{G} 2=104.4 \%$ and Total aflatoxins $=103.3 \%$.

Linearity: Linearity from regression curve was; $\mathrm{B} 1=0.991 ; \mathrm{B} 2=0.997, \mathrm{G} 1=0.994 ; \mathrm{G} 2=0.995$.

Human health risk assessment of exposure to total aflatoxins via consumption of cereals.

Exposure estimation. Calculation of the Estimated Daily Intake (EDI) was done by using the mean levels of aflatoxins obtained in maize samples, the daily intakes of the same samples ${ }^{6}$, and the average body weight. The EDI for mean aflatoxins was calculated according to the following formula and expressed in $\mu \mathrm{g} \mathrm{kg}^{-1}$ of body weight/day $\left(\mu \mathrm{g} \mathrm{kg} \mathrm{bw}{ }^{-1} \mathrm{day}^{-1}\right)^{36}$.

$$
\mathrm{EDI}=\frac{\text { daily intake }(\text { food }) \times \text { mean level of Aflatoxin }}{\text { average bodyweight }}
$$

Daily intake of maize in Ghana according to $\mathrm{MoFA}^{6}$ is $42.5 \mathrm{~kg}$ day $^{-1}$.

Estimation of hazard quotient (HQ). Hazard Quotient (HQ) is otherwise referred to as the Non-Carcinogenic Effect of the toxin. The non-carcinogenic effect of the individual toxin is designated by hazard quotient (HQ) as described by Kortei et al. ${ }^{25}$. The HQ was estimated using Eq. (6): 


$$
\mathrm{HQ}=\frac{\mathrm{EDI}}{\mathrm{RFD}}
$$

where $\mathrm{EDI}$ and $\mathrm{rfD}$ are average daily dose and reference dose respectively.

$$
\mathrm{rfD}=\mathrm{TD}_{50}
$$

Estimation of Hazard Index (HI). The Hazard Index (HI) was calculated according to the below-mentioned formula, by dividing the EDI by $\mathrm{TD}_{50}$, divided by a safety factor of $50,000 . \mathrm{TD}_{50}$ is the dose $\left(\mathrm{ng} \mathrm{kg}^{-1}\right.$ body weight ${ }^{-1}$ day $^{-1}$ ) required to induce tumors in half of the test animals that would have remained tumor-free at zero doses as described by Ismail et al. ${ }^{37}$ and Ishikawa et al. $^{38}$.

$$
H I=\sum_{\mathrm{n}=0}^{1} \frac{\left(\mathrm{EDI} / \mathrm{TD}_{50}\right)}{50000}
$$

Population risk characterization for aflatoxins. Risk characterization for genotoxic and carcinogenic compounds such as aflatoxins is based on the margin of exposures (MOEs), which was calculated by dividing the Benchmark dose lower limit (BMDL) for aflatoxins- $400 \mathrm{ngkg}^{-1}$ bw day $^{-1}$ by toxin exposure ${ }^{39}$

$$
\mathrm{MOE}=\frac{\text { Benchmark dose lower limit }}{\text { EDI (Exposure) }}
$$

In cases where MOEs were lower than 10,000, a public health concern is indicated which implied that aflatoxin exposures above $0.04 \mathrm{ngkg}^{-1}$ bw day ${ }^{-1}$ (as obtained by dividing $400 \mathrm{ngkg}^{-1}$ bw day ${ }^{-1}$ by 10,000 ) represented a risk of public health concern ${ }^{40}$.

Estimated liver cancer risk due to consumption of maize. The estimated liver cancer risk for Ghanaian adult consumers was calculated for aflatoxins because the ingestion of the toxin can be traced to the development of liver cancer ${ }^{11,39}$. This involved estimating the population cancer risk per 100,000 which was obtained by multiplying the EDI value with the average hepatocellular carcinoma (HCC) potency figure from individual potencies of HBsAg-positive and for HBsAg negative groups.

The JECFA estimated potency values for AFB1 which corresponded to 0.3 cancers year ${ }^{-1} 100,000^{-1}$ population $/ \mathrm{ng} \mathrm{kg}^{-1}$ bw day ${ }^{-1}$ (uncertainty range: $0.05-0.5$ ) in $\mathrm{HBs} \mathrm{Ag}$ positive individuals and 0.01 cancers year ${ }^{-1} 100,000^{-1}$ population/ng kg-1 bw day ${ }^{-1}$ (uncertainty range: $0.002-0.03$ ) in HBsAg-negative individuals ${ }^{11,39}$ were adopted for this calculation. Also, the HBsAg + prevalence rate of $10.2 \%$ for Ghana ${ }^{41}$ was adopted and $89.8 \%$ (100-10.2\%) was extrapolated for HBsAg-negative groups. Hence the average potency for cancer in Ghana was estimated as follows:

$$
\begin{aligned}
\text { Average potency } & =(0.3 \times 0.102)+(0.01 \times 0.898) \\
& =0.03958 \text { cancers per year per } 100,000 \text { population per ng Aflatoxins } \mathrm{kg}^{-1} \mathrm{bwday}^{-1}
\end{aligned}
$$

Thus the population risk was estimated using the following formula:

$$
\text { Population risk }=\text { Exposure }(\mathrm{EDI}) \times \text { Average potency }
$$

Statistical analysis. The aflatoxins concentrations were calculated using regression analysis from the curves derived from the standards of the aflatoxins with Excel for Microsoft Windows (version 10). Means and standard deviations of results were subjected to analyses of variance (one-way ANOVA) at the significant difference $(\mathrm{p}<0.05)$ and separation of means were determined via post-hoc test using Duncan's multiple range test DMRT with SPSS 22 (Chicago, USA). Means and standard deviations were computed and graphical representations were used appropriately.

\section{Results}

Good linearity or coefficients of correlations $\left(\mathrm{R}^{2}>0.990\right)$ within the tested range was obtained for most of the food samples tested. For the recovery analysis, one maize and rice samples were previously analyzed to assure the absence of studied mycotoxins, were used in the validation procedure. The Limits of Detection for $\mathrm{AFB}_{1}$ and $\mathrm{AFB}_{2}$ likewise $\mathrm{AFG}_{1}$ and $\mathrm{AFG}_{2}$ ranged between 0.13 and 0.15 while Limits of Quantification ranged between 0.26 and 0.30 respectively for both (Table 2 ).

The number of maize samples contaminated with $\mathrm{AFB} 1, \mathrm{AFB}_{2}, \mathrm{AFG}_{1}, \mathrm{AFG}_{2}$ and $\mathrm{AF}$ Total (Total Aflatoxins) are presented in Tables 3, 4, 5, 6 and 7 . The order of toxicity was $A F B_{1}>A_{F B}>A_{2} G_{1}>A_{2} G_{2}$. As explained by Quinto et al. ${ }^{42}$ the terminal furan moiety of $\mathrm{AFB}_{1}$ is the critical point for determining the degree of biological activity of this group of fungal toxins. Out of a total of ninety (90) samples investigated, 72 (80\%) tested positive for $\mathrm{AFB}_{1}$ and the contamination levels ranged from $0.78 \pm 0.04$ to $339.3 \pm 8.6 \mu \mathrm{g} \mathrm{kg}^{-1}$ for MzNavrongo and MzEjura respectively. For $\mathrm{AFB}_{2}, 59$ (65.5\%) samples tested positive and had levels ranging from $0.52 \pm 0.02$ to $103 \pm 2.5 \mu \mathrm{g} \mathrm{kg}$-1 for MzSefwi-Wiawso and MzEjura respectively. $\mathrm{AFG}_{1}$ was present in 35 (38.8\%) samples of range $0.98 \pm 0.03-14.5 \pm 1.2 \mu \mathrm{g} \mathrm{kg}^{-1}$ for MzDzodze and MzBielepong respectively while, AFG2 was detected in 


\begin{tabular}{|c|c|c|c|c|c|}
\hline \multirow[b]{2}{*}{ Samples } & \multirow[b]{2}{*}{ Total samples } & \multicolumn{2}{|c|}{ Exceeding GSA regulation } & \multicolumn{2}{|c|}{ Exceeding EFSA regulation } \\
\hline & & \begin{tabular}{|l|} 
Yes $(\%)$ \\
\end{tabular} & Range & Yes (\%) & Range \\
\hline \multicolumn{6}{|l|}{ AFTotal } \\
\hline Group 1 & 72 & $41(56.9 \%)$ & $12.12 \pm 1.4-445.01 \pm 8.9$ & $54(75 \%)$ & $4.72 \pm 0.28-445.01 \pm 8.9$ \\
\hline Group 2 & 18 & $3(16.67 \%)$ & $11.26 \pm 1.22-21.66 \pm 0.32$ & $8(44.4 \%)$ & $5.56 \pm 0.26-21.66 \pm 0.32$ \\
\hline Total & 90 & $47(52.2 \%)$ & $11.26 \pm 1.22-445.01 \pm 8.9$ & $62(68.89 \%)$ & $4.72 \pm 0.28-445.01 \pm 8.9$ \\
\hline \multicolumn{6}{|l|}{ AFB1 } \\
\hline Group 1 & 72 & $50(69.44 \%)$ & $5.18 \pm 0.25-339.3 \pm 8.6$ & $56(77.78 \%)$ & $2.2 \pm 0.15-339.3 \pm 8.6$ \\
\hline Group 2 & 18 & $5(27.78 \%)$ & $5.2 \pm 0.5-17.43 \pm 0.81$ & $9(50.0 \%)$ & $2.37 \pm 0.12-16.95 \pm 0.84$ \\
\hline Total & 90 & $55(60.85 \%)$ & $5.18 \pm 0.25-339.3 \pm 8.6$ & $65(72.2 \%)$ & $2.2 \pm 0.15-339.3 \pm 8.6$ \\
\hline
\end{tabular}

Table 8. Proportions of samples that exceeded $\mathrm{AF}_{\mathrm{Total}}$ and $\mathrm{AFB}_{1}$ and limits of Ghana Standard Authority (GSA) and the European Food Safety Authority (EFSA). European Union Food Safety (EFSA) limit for $\mathrm{AF}_{\mathrm{Total}}=4 \mu \mathrm{g} \mathrm{kg}^{-1}$. European Union Food Safety (EFSA) limit for AFB1 $=2 \mu \mathrm{g} \mathrm{kg}^{-1}$. Ghana Standards Authority (GSA) limit $=10 \mu \mathrm{g} \mathrm{kg}^{-1}$. Ghana Standards Authority (GSA) limit $=5 \mu \mathrm{g} \mathrm{kg}{ }^{-1}$.

only 14 (15.5\%) samples, and their values ranged between $1.09 \pm 0.03-5.51 \pm 0.26 \mu \mathrm{g} \mathrm{kg}^{-1}$ for MzLawra and MzOffinso respectively. Lastly, a total ranged between $0.78 \pm 0.04-445.01 \pm 8.9 \mu \mathrm{g} \mathrm{kg}^{-1}$ for MzNavrongo and MzEjura respectively. The total aflatoxin determinations were obtained from $72(80.2 \%)$ samples.

The greatest aflatoxin yields of $445.01 \pm 8.9$ and $339.3 \pm 8.6 \mu \mathrm{g} \mathrm{kg}^{-1}$ for $\mathrm{AF}_{\text {Total }}$ and $\mathrm{AFB}_{1}$ respectively, were significantly $(\mathrm{p}<0.05)$ higher than all other samples studied in the various categories.

Toxin quantity limits prescribed by the Ghana Standards Authority which are slightly higher and more flexible than the European Union (EFSA) are 5, $10 \mu \mathrm{g} \mathrm{kg}$-1 and 2, $4 \mu \mathrm{g} \mathrm{kg}^{-1}$ respectively for $\mathrm{AFB}_{1}$ and Total aflatoxins.

Results from the study were compared to the European Food Safety Authority (EFSA) and Ghana Standards Authority (GSA) regulatory concentration limits for total aflatoxins (AF Total) and Aflatoxins $\mathrm{B}_{1}\left(\mathrm{AFB}_{1}\right)$. From Table 8, a majority of $41 / 72(56.9 \%)$ of the 72 samples analyzed for total aflatoxins in group one (white maize samples) exceeded the limits of GSA. These maize samples had $\mathrm{AF}_{\text {Total }}$ ranging from $12.12 \pm 1.4$ to $445.01 \pm 8.9 \mu \mathrm{kg}^{-1}$. Only $3 / 18$ (16.67\%) out of colored maize samples (Group two) was found to exceed the GSA limit which also ranged $11.26 \pm 1.22-21.66 \pm 0.32 \mu \mathrm{g} \mathrm{kg}{ }^{-1}$.

Overall, $47 / 90(52.2 \%)$ of the maize recorded values above the set limit and ranged $11.26 \pm 1.22-445.01 \pm 8$ $.9 \mu \mathrm{g} \mathrm{kg}-1$.

About 54/72 (75\%) of white maize and 8/18 (44.4\%) colored maize corresponding to ranges of $4.72 \pm 0.28-445.01 \pm 8.9$ and $5.56 \pm 0.26-21.66 \pm 0.32 \mu \mathrm{g} \mathrm{kg}^{-1}$ respectively exceeded the tolerable limit of the EFSA. Overall for EFSA, $62 / 90(68.89 \%)$ of white maize samples of range $4.72 \pm 0.28-445.01 \pm 8.9 \mu \mathrm{g} \mathrm{kg}^{-1}$ exceeding limits were recorded. Whereas 70.4 and $11.5 \%$ of samples respectively from the two groups exceeded EFSA for $\mathrm{AFB}_{1}$ (Table 8).

For $\mathrm{AFB}_{1}$, GSA limits exceeded were 50/72 (69.44\%) and ranged 5.18 $\pm 0.25-339.3 \pm 8.6 \mu \mathrm{g} \mathrm{kg}^{-1}$ while for the colored maize samples, $5 / 18(27.78 \%)$ of range $5.2 \pm 0.5-17.43 \pm 0.81 \mu \mathrm{g} \mathrm{kg}^{-1}$ were recorded. For the overall, $55 / 90(60.85 \%)$ of range, $5.18 \pm 0.25-339.3 \pm 8.6 \mu \mathrm{g} \mathrm{kg}^{-1}$ were recorded.

Values of $56 / 72(77.78 \%)$ with range $2.2 \pm 0.15-339.3 \pm 8.6 \mu \mathrm{g} \mathrm{kg}^{-1}$ and $9 / 18(50.0 \%)$ with range $2.37 \pm 0.12-1$ $6.95 \pm 0.84 \mu \mathrm{g} \mathrm{kg}-1$ were recorded for white and colored maize samples respectively. Finally, as the overall, $65 / 90$ $(72.2 \%)$ maize samples of range $2.2 \pm 0.15-339.3 \pm 8.6 \mu \mathrm{g} \mathrm{kg}^{-1}$ exceeded limits for EFSA.

Risk assessment. The Estimated Daily Intakes (EDI) of the total aflatoxins in the maize samples were 109.7, 58.8, 33.08 and $25.2 \mu \mathrm{g} \mathrm{Kg} \mathrm{bw}{ }^{-1}$ day $^{-1}$ for infants, children, adolescents, and adults respectively. For the Hazard Quotient (HQ), values of $5.5 \times 10^{5}, 2.94 \times 10^{5}, 1.65 \times 10^{5}$, and $1.26 \times 10^{5}$ were recorded respectively. A range of 2.5-10.97 was recorded for H.I. Margin of Exposure (MOE) values recorded were 3.64, 6.80, 12.09 and 6.75

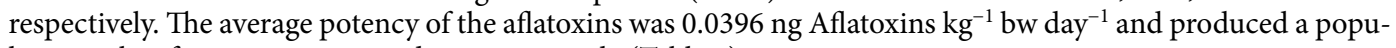
lation risks of $4.344,2.3,1.31$ and 1.0 respectively (Table 9).

For $\mathrm{AFB}_{1}$, the EDIs for infants, children, adolescents, and adults were 84.5, 45.5, 25.6 and $19.5 \mu \mathrm{g} \mathrm{Kg} \mathrm{bw}{ }^{-1}$ day $^{-1}$ respectively. HQ values recorded were $5.5 \times 10^{5}, 2.94 \times 10^{5}, 1.65 \times 10^{5}$ and $1.26 \times 10^{5}$ respectively. Margin of Exposure (MOE) values recorded were $4.73,8.79,15.63$ and 20.51 respectively. The average potency was the same as total aflatoxins while the population risks were respectively $3.35,1.80,1.01$ and 0.77 (Table 10).

\section{Discussion}

Aflatoxin contamination of market maize is a significant public health concern. Our findings demonstrated widespread aflatoxin contamination of maize within the regional market distribution system. The different maize samples obtained from the different markets across the country had varying quantities of AFB1 and AFtotal. The greatest quantity of aflatoxins recorded from this study $\left(445.01 \pm 8.9 \mu \mathrm{g} \mathrm{kg}^{-1}\right)$ was, by and large, lower than values of 692 and $945 \mathrm{ng} \mathrm{g}^{-1}$ from maize samples obtained from Fumesua and Ejura in Ghana respectively by Dadzie et al. ${ }^{24}$.

$\mathrm{Kpodo}^{50}$ in earlier surveys reported aflatoxin levels in the range of $20-355 \mathrm{ng} \mathrm{g}^{-1}$ maize samples from silos and warehouses in Ejura while fermented corn dough collected from major processing sites also contained aflatoxin 


\begin{tabular}{|c|c|c|c|c|c|c|c|c|}
\hline & Av.body wgt.(kg) & References (weight) & $\begin{array}{l}\text { Estimated daily } \\
\text { intake (EDI) } \\
\left(\mu \mathrm{gg} \mathrm{bw}^{-1} \mathrm{day}^{-1}\right)\end{array}$ & $\begin{array}{l}\text { Hazard quotient } \\
\text { (H.Q) }\end{array}$ & hazard index (h.i) & MOE & $\begin{array}{l}\text { Av. potency } \\
\text { (ng Aflatoxins } \\
\mathrm{kg}^{-1} \text { bw day }{ }^{-1} \text { ) }\end{array}$ & Population risk \\
\hline Infants (6-52mths) & 7 & $\begin{array}{l}\text { Glover-Amengor } \\
\text { et al. }{ }^{43} \\
\text { Abeshu et al. }{ }^{44}\end{array}$ & 109.7 & $5.5 \times 10^{5}$ & 10.97 & 3.64 & 0.0396 & 4.344 \\
\hline $\begin{array}{l}\text { Children } \\
\text { (5-11 years) }\end{array}$ & $26(24-28)$ & $\begin{array}{l}\text { Biritwum et al. }{ }^{45} \\
\mathrm{WHO}^{46}\end{array}$ & 58.8 & $2.94 \times 10^{5}$ & 5.88 & 6.80 & 0.0396 & 2.3 \\
\hline $\begin{array}{l}\text { Adolescents } \\
\text { (12-18 years) }\end{array}$ & $46.25(38.5-54)$ & Afrifa-Anane et al. ${ }^{47}$ & 33.08 & $1.65 \times 10^{5}$ & 3.30 & 12.09 & 0.0396 & 1.31 \\
\hline Adults (18-60 years) & 60.7 & Walpole et al. ${ }^{48}$ & 25.2 & $1.26 \times 10^{5}$ & 15.87 & 6.75 & 0.0396 & 1.0 \\
\hline
\end{tabular}

Table 9. Evaluation of risk for Total Aflatoxins via consumption of maize. Margin of Exposure-MOE. ${ }^{\star}$ Mean aflatoxins-36.13 $\mu \mathrm{g} \mathrm{kg}^{-1}$. ${ }^{\star}$ Daily intake of maize for infants was halved $(0.5 \times 42.5 \mathrm{~kg})$. ${ }^{\star}$ Daily intake of $42.5 \mathrm{~kg}$ was used for children, adolescents, and adults. $\mathrm{TD}_{50}=0.2 \mathrm{ng} \mathrm{Kg} \mathrm{bw}{ }^{-149} .1 \mathrm{ng}=0.001 \mu \mathrm{g}$.

\begin{tabular}{|c|c|c|c|c|c|c|c|c|}
\hline & $\begin{array}{l}\text { Av.Body weight } \\
\text { (kg) }\end{array}$ & $\begin{array}{l}\text { References } \\
\text { (weight) }\end{array}$ & $\begin{array}{l}\text { Estimated daily } \\
\text { intake (EDI) } \\
\left(\mu \mathrm{g} \mathrm{Kg} \mathrm{bw}{ }^{-1} \text { day }^{-1}\right)\end{array}$ & $\begin{array}{l}\text { Hazard quotient } \\
\text { (H.Q) }\end{array}$ & Hazard Index (H.I) & MOE & $\begin{array}{l}\text { Av. potency } \\
\text { (Aflatoxins } \mathrm{ng} \mathrm{kg}^{-1} \\
\text { Bw day } \text { dal }^{-1}\end{array}$ & Population risk \\
\hline Infants (6-52mths) & $7.08(2.5-11.65)$ & $\begin{array}{l}\text { Glover-Amengor } \\
\text { et al. }{ }^{43} \\
\text { Abeshu et al. }{ }^{44}\end{array}$ & 84.5 & $4.2 \times 10^{5}$ & 8.5 & 4.733 & 0.0396 & 3.35 \\
\hline $\begin{array}{l}\text { Children } \\
\text { (5-11 years) }\end{array}$ & $26(24-28)$ & $\begin{array}{l}\text { Biritwum et al. }{ }^{45} \\
\text { WHO }^{46}\end{array}$ & 45.5 & $2.3 \times 10^{-3}$ & $4.6 \times 10^{-3}$ & 8.79 & 0.0396 & 1.80 \\
\hline $\begin{array}{l}\text { Adolescents } \\
\text { (12-18 years) }\end{array}$ & $46.25(38.5-54)$ & Afrifa-Anane et al. ${ }^{47}$ & 25.6 & $1.3 \times 10^{5}$ & 2.6 & 15.63 & 0.0396 & 1.01 \\
\hline $\begin{array}{l}\text { Adults }(18- \\
60 \text { years })\end{array}$ & 60.7 & Walpole et al. ${ }^{48}$ & 19.5 & $9.7 \times 10^{4}$ & 1.95 & 20.51 & 0.0396 & 0.77 \\
\hline
\end{tabular}

Table 10. Evaluation of risk for Aflatoxins B1 (AFB1) via consumption of maize. The margin of ExposureMOE. TD $_{50}=0.2 \mathrm{ng} \mathrm{kg} \mathrm{bw}{ }^{-149} .1 \mathrm{ng}=0.001 \mu \mathrm{g}$. ${ }^{\star}$ Mean of AFB1 $27.85 \mu \mathrm{g} \mathrm{kg}^{-1}$. ${ }^{\star}$ Daily intake of maize for infants was halved $(0.5 \times 42.5)$. ${ }^{\star}$ Daily intake of $42.5 \mathrm{~kg}$ was used for children, adolescents, and adults.

levels of 0.7-313 $\mathrm{ng} \mathrm{g}^{-1}$. James et al. ${ }^{51}$ also found high average aflatoxin levels in maize samples collected from North Kwahu (153 $\left.\mathrm{ng} \mathrm{g}^{-1}\right)$, Ejura Sekyere-Dumasi (121 ng g $\left.{ }^{-1}\right)$ and Nkoranza (134 ng g $\left.{ }^{-1}\right)$.

Agbetiameh et al. ${ }^{26}$ reported values of range 1-341 ppb in maize from different ecological zones in Ghana. Likewise, Blankson et al. ${ }^{28}$ also reported a range of $1.77 \pm 0.01-24.58 \pm 0.05 \mu \mathrm{g} \mathrm{kg}^{-1}$ in maize-based samples in locally prepared cereals for consumption in Ghana.

From Kenya, Nduti et al. $^{52}$ reported values of range $7.92 \pm 1.57-22.54 \pm 4.94 \mathrm{ppb}$ from maize flour samples obtained from three regions.

Lewis et al. ${ }^{8}$ reported greater values of aflatoxin quantities of $>1000 \mathrm{ppb}$ from maize samples as they investigated aflatoxin contamination of commercial maize products during an outbreak of acute aflatoxicosis in Eastern and Central Kenya.

AFB1 was present in seven of eight samples ranging from 30 to $6127 \mu \mathrm{g} \mathrm{kg}{ }^{-1}$ and two of eight samples were found positive for AFB2 ranging from 53 to $1738 \mu \mathrm{g} \mathrm{kg}-1$ as reported by Sewram et al. ${ }^{53}$ from Brazil as they investigated corn-based infant food sold on different markets. In their study in Ghana, aflatoxins were reported to markedly contaminate local food (weanimix) from cereal-legume blend for children and reported aflatoxin B1 levels in local weanimix of range 7.9-500 $\mathrm{ppb}\left(\mathrm{ug} \mathrm{kg}^{-1}\right)^{54}$.

The reasonably high levels of aflatoxins detected in this study, put forward that aflatoxins can continue to persist in food even after the inactivation of the fungi in spite of all the rigorous processing methods because of their ability to resist chemical and thermal changes ${ }^{55}$. A danger looms hereafter, since there is a high likelihood of detecting aflatoxins in processed cereal foods more so if the ingredients used for the food are initially contaminated before processing and subsequent consumption. Thus, the incidence of aflatoxins in processed cereal-based food might indicate contamination of the raw cereals at a point in the value chain (either on the farm or during storage). It is also noteworthy that the not detected (n.d) status recorded in some samples may not necessarily mean total absence of aflatoxins but were simply below detectable limits of the equipment.

Aflatoxins contamination occurs via an initial phase during crop development and a second phase during crop maturation. The contamination is greater in warm, humid, and even hot deserts and drought conditions ${ }^{56}$ since mycotoxins are optimally produced in adverse periods.

Atoxigenic biocontrol of aflatoxins promises a safe, economical, ecosystem friendly, cost-effective method of aflatoxin mitigation throughout the value chain ${ }^{57-61}$. Implementing aflatoxin biocontrol management strategies to reduce aflatoxin contamination in the field and throughout storage would result in improved health, enhanced trade, increased income, and the welfare of farmers and consumers in Ghana.

Climatic variations of the different agro-ecological zones might be an explanation to the seasonal occurrences of types and levels of toxins in the food material ${ }^{62}$. Planning interventions against dietary exposure to aflatoxins 
form an important basis for these current findings since the rainfall pattern in major parts of the country is categorized bimodally by long and short rainy seasons intermingled with brief dry spells ${ }^{62}$. A general mycotoxicosis risk is signaled through a seasonal assessment of aflatoxin residues in food specifically given climatic changes triggering hot and dry influences linked to increased contamination ${ }^{56,63,64}$. It was also observed in this study that colored maize recorded lower quantities of aflatoxins as compared with levels of the white maize samples. Presumably, the biochemical substances present in the maize accounting for the colorations such as beta carotene, zeaxanthins, lutein etc. may have inhibited the propagation of Aspergillus species.

In Africa, of which Ghana is no exception, smallholder farmers produce most of the maize of which most of them use poor harvest techniques such as insufficient drying and storage of their crops. Additionally, the cultivation of local maize varieties which are susceptible to both insect damage and diseases, less drought-tolerant, expose the maize to infection by encouraging the growth of aflatoxin-producing fungi during crop development, maturation and harvest in the field ${ }^{65}$.

Despite the availability of improved varieties, local varieties are still planted by a significant fraction of maize farmers. Even though there is knowledge of the occurrence of aflatoxin accumulation in maize collected in markets and farmer stores across Ghana ${ }^{26}$, little is known of the aflatoxin levels when the maize is still in the field (prior-to-harvest maize) and the composition of community structures of Aspergillus section flavor associated with the maize in Ghana.

\section{Conclusion}

It can be surmised from the results of this study that from a total of ninety (90) maize samples investigated, $65(72.2 \%)$ tested positive for $\mathrm{AFB}_{1}$ and ranged from $2.2 \pm 0.15-339.3 \pm 8.6 \mu \mathrm{g} \mathrm{kg}^{-1}$. A similar proportion of 62 $(68.89 \%)$ was also recorded for total aflatoxins $\left(\mathrm{AF}_{\text {Total }}\right)$ and ranged between $4.72 \pm 0.28-445.01 \pm 8.9 \mu \mathrm{g} \mathrm{kg}^{-1}$ and these were above the limits set by the Ghana Standards Authority (GSA) (5 and 10-15 $\mu \mathrm{g} \mathrm{kg}^{-1}$ ) and the European Food Safety Authority (EFSA) ( 2 and $\left.4 \mu \mathrm{g} \mathrm{kg}^{-1}\right)$. Human health risk assessment from aflatoxins exposure through maize consumption from the markets by infants, children, adolescents, and adults showed a significant non-carcinogenic adverse health risk to humans since all calculated values for Hazard Quotient (HQ) were $>1$ while there was no observed adverse health effect $(\mathrm{HI}<1)$. Conversely, MOE values obtained in this study were all greater than $0.04 \mathrm{ngkg}^{-1} \mathrm{bw}$ day $^{-1}$ which implied a potential health risk as suggested by the World Health Organization (2008).

Government institutions, private and non-governmental organizations, as well as national media networks need to play a key role in raising consciousness of the public health impacts of aflatoxin. Bearing in mind the necessity of attaining food security and food safety for vulnerable people in these areas, there is also a need for data and risk management capacity tools for locally driven policy reform.

Limitations of the study. The human health risk assessments were arrived at using deterministic methods (based on assumptions) as opposed to using probabilistic approaches.

The primary data obtained from the laboratory, are usually presented as means + standard deviation from three determinations which statistically, do not represent the true average of the concentrations.

Received: 16 January 2021; Accepted: 1 February 2021

Published online: 22 February 2021

\section{References}

1. Lewis, L. et al. Aflatoxin contamination of commercial maize products during an outbreak of acute aflatoxicosis in Eastern and Central Kenya. J. Environ. Health Perspect. 113(12), 1763-1767. https://doi.org/10.1289/ehp.7998 (2005).

2. Mohajan, H. K. Food and nutrition scenario of Kenya. Am. J. Food Nutr. 2(2), 28-38 (2014).

3. Matumba, L. et al. Uncommon occurrence ratios of aflatoxin B1, B2, G1, and G2 in maize and groundnut from Malawi. Mycotoxin Res. 31, 57-62 (2015).

4. Shephard, G. S., Rheeder, J. P. \& van der Westhuizen, L. Effect of the traditional cooking practice on fumonisin content of maize porridge consumed in the former Transkei region of South Africa. World Mycotoxin J. 5(4), 405-407 (2012).

5. Hove, M. et al. Occurrence and risk assessment of mycotoxins in subsistence farmed maize from Zimbabwe. Food Control 69, 36-44. https://doi.org/10.1016/j.foodcont.2016.04.038 (2016).

6. Quiñones, E. J. \& Diao, X. Assessing Crop Production and Input Use Patterns in Ghana-What Can We Learn from the Ghana Living Standards Survey (GLSS5); Ghana Strategy Support Program (GSSP) Working Paper No. 0024. (International Food Policy Research Institute: Accra, Ghana, 2011).

7. Food Balance Sheets-FAOSTAT. http://www.fao.org/faostat/en/\#data/FBS/visualize (2018/2019).

8. MoFA. Food and Agriculture Sector Development Policy (FASDEP I) 55 (2000).

9. Kimanya, M. E. et al. Co-occurrence of fumonisins with aflatoxins in home-stored maize for human consumption in rural villages of Tanzania. Food Addit. Contam. 25, 1353-1364 (2008).

10. Mutegi, C. et al. Market attributes and their effect on levels of aflatoxin in peanuts (Arachis hypogaea L.) from Nairobi and western Kenya. East Afr. Agric. For. J 77, 95-103 (2010).

11. Shephard, G. S. Risk assessment of aflatoxins in food in Africa. AFood Addit. Contam. A 25(10), 1246-1256 (2008).

12. Frisvad, J. C., Thrane, U., Samson, R. A. \& Pitt, J. I. Important mycotoxins and the fungi which produce them. In Advances in Food Mycology Advances in Experimental Medicine and Biology (eds Hocking, A. D. et al.) (Springer Science and Business Media, New York, 2006).

13. Schuda, P. F. Aflatoxin chemistry and syntheses. In Syntheses of Natural Products 75-111 (Springer, Berlin, 1980).

14. Mahato, D. K. et al. Aflatoxins in food and feed: An overview on prevalence detection and control strategies. Front. Microbiol. 10, 2266. https://doi.org/10.3389/fmicb.2019.02266 (2019).

15. Kumar, P., Mahato, D. K., Kamle, M., Mohanta, T. K. \& Kang, S. G. Aflatoxins: A global concern for food safety, human health, and their management. Front. Microbiol. 7, 2170. https://doi.org/10.3389/fmicb.2016.02170 (2017).

16. Yu, J., Bhatnagar, D. \& Ehrlich, K. C. Aflatoxin biosynthesis. Rev. Iberoam. Micol. 19, 191-200 (2002). 
17. Yabe, K. \& Nakajima, H. Enzyme reactions and genes in aflatoxin biosynthesis. Appl. Microbiol. Biotechnol. 64, 745-755. https:// doi.org/10.1007/s00253-004-1566-x (2004).

18. Kimanya, M. E. The health impacts of mycotoxins in the eastern Africa region. Curr. Opin. Food Sci. 6, 7-11 (2015).

19. Food and Agriculture Organization of the United Nations (FAO) (2017). Crop Prospects and Food Situation. GIEWS Reports http:// www.fao.org/3/a-i8278e.pdf. Accessed 17/07/2020.

20. Odamtten, G. T. Natural Occurrence, Economic Impact and Control of Aflatoxins in Africa (WHO Expert Committee Meeting on Aflatoxins and Health, Brazaville, 2005).

21. Awuah, R. T., Agyemang, K. O., Fialor, S. C. \& Jolly, C. M. Are Ghanaians aware of the aflatoxin menace? In Mycotoxins: Detection Methods, Management, Public Health, and Agricultural Trade (eds Leslie, J. F. et al.) 327-334 (CABI Publishing, Wallingford, 2008).

22. Kpodo, K. A. \& Bankole, S. A. Mycotoxin contamination in foods in West and Central Africa. In Mycotoxins: Detection Methods, Management, Public Health and Agricultural Trade 103-116 (CABI Publishing, Wallingford, 2008).

23. Achaglinkame, M. A., Opoku, N. \& Amagloh, F. K. Aflatoxin contamination in cereals and legumes to reconsider usage as complimentary food ingredients for Ghanaian infants: A review. J. Nutr. Intermed. Metab. 10, 1-7. https://doi.org/10.1016/j. jnim.2017.09.001 (2017).

24. Dadzie, M. A. et al. Distribution of Aspergillus flavus and aflatoxin accumulation in stored maize grains across three agro-ecologies in Ghana. Food Control 104, 91-98. https://doi.org/10.1016/j.foodcont.2019.04.035 (2019).

25. Kortei, N. K., Agyekum, A. A., Akuamoa, F., Kyei-Baffour, V. \& Alidu, H. W. Risk assessment and levels of naturally occurring aflatoxins in packaged cereals and cereal-based foods on the Ghanaian market. Toxicol. Rep. 6, 34-41. https://doi.org/10.1016/j. toxrep.2018.11.012 (2019).

26. Agbetiameh, D. et al. Prevalence of aflatoxin contamination in maize and groundnut in Ghana: Population structure, distribution, and toxigenicity of the causal agents. Plant Dis. 102, 764-772 (2018).

27. Ofosu, I. W. et al. Aflatoxin exposures and risks in maize-based foods consumed by university students. Acta Sci. Nutr. Health 3(2), 110-119 (2019).

28. Blankson, G. K., Mills-Robertson, F. C. \& Ofosu, I. W. Survey of occurrence levels of Aflatoxins in selected locally processed cereal-based foods for human consumption from Ghana. Food Control 95, 170-175. https://doi.org/10.1016/j.foodcont.2018.08.005 (2019).

29. GSA. Ghana Intensifies Bid for the Eradication of Aflatoxins. https://www.gsa.gov.gh/2019/04/ghana-intensifies-bid-for-eradicatio n-of-aflatoxin-related-losses-from-agricultural-value-chains/ (2019)

30. MOFA (Ministry of Food and Agriculture). Agriculture in Ghana. Facts and Figures (Statistics, Research and Information Directorate (SRID), MoFA, Accra, 2014).

31. Nkrumah, F. et al. Rainfall variability over Ghana: Model versus rain gauge observation. Int. J. Geosci. 5(7), 673-683. https://doi. org/10.4236/ijg.2014.57060 (2014).

32. Ghana Statistical Service. 2010 population and housing census, 2010 Popul. Hous. Census Natl. Anal. Rep., 305-337 (2013).

33. Abbam, T., Johnson, F. A., Dash, J. \& Padmadas, S. S. Spatiotemporal variations in rainfall and temperature in Ghana over the twentieth century, 1900-2014. Earth Space Sci. 5, 120-132. https://doi.org/10.1002/2017EA000327 (2018).

34. European Committee for Standardization (CEN). Foodstuffs: Determination of Aflatoxin B1 and the Sum of Aflatoxin B1, B2, G1 and G2 in Hazelnuts, Peanuts, Pistachios, Figs, and Paprika Powder: High Performance Liquid Chromatographic Method with Postcolumn Derivatisation and Immunoaffinity Column Cleanup. https://www.cen.eu/cen/Sectors/TechnicalCommitteesWorkshops/ CENTechnicalCommittees/Pages/Standards.aspx?param=6256\&title=CEN/TC\%20275 (2007).

35. Kortei, N. K. et al. Aflatoxins in randomly selected groundnuts (Arachis hypogaea) and its products from some local markets across Ghana: Human risk assessment and monitoring. Toxicol. Rep. 8, 186-195. https://doi.org/10.1016/j.toxrep.2021.01.002 (2021).

36. Dos Santos, J. S. et al. Natural occurrence of deoxynivalenol in wheat from Paraná State Brazil and estimated daily intake by wheat products. Food Chem. 138, 90-95 (2013).

37. Ismail, A. et al. Seasonal prevalence level of aflatoxin M1 and its estimated daily intake in Pakistan. Food Control 60, 461-465 (2016).

38. Ishikawa, A. T. et al. Exposure assessment of infants to Aflatoxin M1 through consumption of breast milk and infant powdered milk in Brazil. Toxins 8, 246 (2016).

39. EFSA European Food Safety Authority. Opinion of the scientific panel on contaminants in the food chain on a request from the commission related to the potential increase of consumer health risk by a possible increase of the existing maximum levels for aflatoxins in almonds, hazelnuts, and pistachios, and derived products. EFSA J. 446, 1-127 (2007).

40. WHO. http://www.who.int/healthinfo/globalburdendisease/2004reportupdate/en/index.html (2008).

41. Ofori-Asenso, R. \& Agyeman, A. A. Hepatitis B in Ghana: A systematic review\& meta-analysis of prevalence studies (1995-2015). BMC Infect. Dis. 16, 130. https://doi.org/10.1186/s12879-016-1467-5 (2016).

42. Quinto, M., Spadaccino, G., Palermo, C. \& Centonze, D. Determination of aflatoxins in cereal flours by solid-phase microextraction coupled with liquid chromatography and post-column photochemical derivatization-fluorescence detection. J. Chromatogr. A 1216, 8636-8641 (2009).

43. Glover-Amengor, M. et al. Nutritional status of children 0-59 months in selected intervention communities in northern Ghana from the Africa RISING project in 2012. Arch. Public Health 74, 12. https://doi.org/10.1186/s13690-016-0124-1 (2016).

44. Abeshu, M. A., Lelisa, A. \& Geleta, B. Complementary Feeding: Review of recommendations, feeding practices, and adequacy of homemade complementary food preparations in developing countries: Lessons from Ethiopia. Front. Nutr. 3, 41. https://doi. org/10.3389/fnut.2016.00041 (2016).

45. Biritwum, R. B., Gyapong, J. \& Mensah, G. The epidemiology of Obesity in Ghana. Ghana Med. J. 39(3), 82-85 (2005).

46. WHO. WHO Child Growth Standards: Length/Height-for-Age, Weight-for-Age, Weight-for-Length, Weight-for-Height and Body Mass Index-for-Age: Methods and Development. World Health Organization. "The global burden of disease: 2004 update," 2008 (2006). https://www.who.int/childgrowth/standards/Technical_report.pdf (2006)

47. Afrifa-Anane, E., Agyemang, C., Codjoe, N. A., Ogedegbe, G. \& De-Graft Aikins, A. The association of physical activity, body mass index and the blood pressure levels among urban poor youth in Accra, Ghana. BMC Public Health 15(269), 1-9. https://doi. org/10.1186/s12889-015-1546-3 (2015).

48. Walpole, S. C. et al. The weight of nations: an estimation of adult human biomass. BMC Public Health 12, 439 (2012).

49. Kuiper-Goodman, T. Uncertainties in the risk assessment of three mycotoxins: Aflatoxin, ochratoxin, and zearalenone. Can. J. Physiol. Pharmacol. 68, 1017-1024 (1990).

50. Kpodo, K. A., Sørensen, A. \& Jakobsen, M. The occurrence of mycotoxins in fermented maize products. Food Chem. 56, 147-153 (1996).

51. James, B. et al. Public information campaign on aflatoxin contamination of maize grains in market stores in Benin, Ghana and Togo. Food Addit. Contam. B Surveill. 24(11), 1283-2129. https://doi.org/10.1080/02652030701416558 (2007).

52. Nduti, N. N., Njeru, P. N., Mwaniki, M. \& Reid, G. Aflatoxin variations in maize flour and grains collected from various regions of Kenya. Afr. J. Food Agric. Nutr. Dev. 17(1), 11743-11756. https://doi.org/10.18697/ajfand.77.16875 (2017).

53. Sewram, V., Shephard, G. S., Marasas, W. F. O. \& Castro, M. F. Improving extraction of Fumonisin mycotoxins from Brazilian corn-based infant foods. J. Food Prot. 66(5), 854-859 (2003).

54. Kumi, J. et al. Aflatoxins and fumonisins contamination of home-made food (weanimix) from cereal-legume blends for children. Ghana Med. J. 3, 48 (2014). 
55. Yazdanpanah, H., Mohammadi, T., Abouhossain, G. \& Cheraghali, A. M. Effect of roasting on degradation of aflatoxins in contaminated pistachio nuts. Food Chem. Toxicol. 43, 1135-1139 (2005).

56. Cotty, P. J. \& Jaime-Garcia, R. Influences of climate on aflatoxin producing fungi and aflatoxin contamination. Int. J. Food Microbiol. 119, 109-115. https://doi.org/10.1016/j.ijfoodmicro.2007.07.060 (2007).

57. Atehnkeng, J., Ojiambo, P. S., Cotty, P. J. \& Bandyopadhyay, R. Field efficacy of a mixture of toxigenic Aspergillus flavus Link: Fr vegetative compatibility groups in preventing aflatoxin contamination in maize (Zea mays L.). Biol. Control 72, 62-70. https://doi. org/10.1016/j.biocontrol.2014.02.009 (2014).

58. Wu, F., Liu, Y. \& Bhatnagar, D. Cost-effectiveness of aflatoxin control methods: Economic incentives. Toxin Rev. 27, 203-225. https ://doi.org/10.1080/15569540802393690 (2008).

59. Mehl, H. L. et al. Aspergillus flavus diversity on crops and in the environment can be exploited to reduce aflatoxin exposure and improve health. Ann. N. Y. Acad. Sci. 1273, 7-17. https://doi.org/10.1111/j.1749-6632.2012.06800.x (2012)

60. Bandyopadhyay, R. et al. Biological control of aflatoxins in Africa: current status and potential challenges in the face of climate change. World Mycotoxin J. 9, 771-789. https://doi.org/10.3920/wmj2016.2130 (2016).

61. Bandyopadhyay, R. et al. "Ground-truthing" efficacy of biological control for aflatoxin mitigation in farmers fields in Nigeria: From field trials to commercial usage. Front. Microbiol. https://doi.org/10.3389/fmicb.2019.02528 (2019).

62. Mugo, R. M. J., Ininda, M. \& Okoola, R. E. Interannual variability of onset and cessation of the long rains in Kenya. J. Meteorol. Relat. Sci. 9, 30-47 (2016).

63. Kebede, H., Abbas, H. K., Fisher, D. K. \& Bellaloui, N. Relationship between aflatoxin contamination and physiological responses of corn plants under drought and heat stress. Toxins 4(11), 1385-1403 (2012).

64. Smith, L. E. et al. Examining environmental drivers of spatial variability in aflatoxin accumulation in Kenyan maize: Potential utility in risk prediction models. Afr. J. Food Agric. Nutr. Dev. 16(3), 11086-11105 (2016).

65. Sserumaga, J. P., Ortega- Beltran, A., Wagacha, J. M., Mutegi, C. K. \& Bandyopadhyay, R. Aflatoxin-producing fungi associated with pre-harvest maize contamination in Uganda. Int. J. Food Microbiol. 313, 108376. https://doi.org/10.1016/j.ijfoodmicro.2019.10837 $6(2020)$.

\section{Acknowledgements}

Authors are grateful to the University of Health and Allied Sciences and the CSIR-Food Research Institute for their immense support and the successful completion of this research work. Our heartfelt thanks to the families of the authors for their undying support.

\section{Author contributions}

N.K.K, T.A, P.T.A, and R.A.S contributed to the conception and design of the experiment, analysis of the data and writing the manuscript. N.K.K., F.A., P.G.A, P.E.A and T.A. did the sample collection. V.K-B. and H.A.A. analyzed the samples.

\section{Competing interests}

The authors declare no competing interests.

\section{Additional information}

Correspondence and requests for materials should be addressed to N.K.K.

Reprints and permissions information is available at www.nature.com/reprints.

Publisher's note Springer Nature remains neutral with regard to jurisdictional claims in published maps and institutional affiliations.

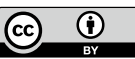

Open Access This article is licensed under a Creative Commons Attribution 4.0 International License, which permits use, sharing, adaptation, distribution and reproduction in any medium or format, as long as you give appropriate credit to the original author(s) and the source, provide a link to the Creative Commons licence, and indicate if changes were made. The images or other third party material in this article are included in the article's Creative Commons licence, unless indicated otherwise in a credit line to the material. If material is not included in the article's Creative Commons licence and your intended use is not permitted by statutory regulation or exceeds the permitted use, you will need to obtain permission directly from the copyright holder. To view a copy of this licence, visit http://creativecommons.org/licenses/by/4.0/.

(C) The Author(s) 2021 